\title{
Osteomyelitis management: More art than science?
}

\author{
BL Johnston $M D^{1}$, JM Conly $M D^{2}$
}

$\mathrm{O}$ ver the past 40 years, several authoritative reviews on osteomyelitis have been published in The New England Journal of Medicine (1-4). They have provided commentary on definition, pathogenesis, diagnosis and treatment. Simplistically, acute osteomyelitis is a newly recognized bone infection, and chronic osteomyelitis is the relapse of a previously treated or untreated infection (4). One hallmark of chronic osteomyelitis is necrotic bone, resulting from impaired blood flow related to raised intraosseous pressure (4). There are several staging systems for osteomyelitis to help guide therapeutic decisions and allow for comparisons across studies. The oldest of these is Waldvogel's staging system, which classifies osteomyelitis as hematogenous or contiguous, with contiguous further subdivided into the presence or absence of vascular insufficiency (5). This is probably the classification system most familiar to infectious diseases physicians, and it provides a framework for treatment recommendations in Mandell's Principles and Practice of Infectious Diseases (6) and The Sanford Guide to Antimicrobial Therapy 2006 (7). Another commonly applied staging system for long-bone osteomyelitis is the Cierny-Mader staging system, which is based on anatomy, physiological status of the host (normal or compromised), and systemic or local factors that affect immune surveillance, metabolism and bone vascularity $(5,8)$. While the CiernyMader staging system is more detailed than the Waldvogel staging system, there are similarities in the anatomical stratification, with anatomical stage 1 (medullary) correlating with hematogenous, and anatomical stages two to four (superficial, localized and diffuse) related to contiguous, although the Cierny-Mader system better describes the extent of disease and surgical management. Integrating the anatomical and host factors in the Cierny-Mader system creates 12 potential clinical stages (9). Most cases of osteomyelitis in adults are contiguous, and the usual antecedent factors are trauma, surgery or pressure ulcers. Hematogenous osteomyelitis, when it occurs in adults, involves primarily the axial skeleton (10). Most of the other staging systems relate to anatomical and pathological features of the osteomyelitis, such as presence of sinuses, fracture (united or nonunion), duration of drainage and degree of bone loss (8). Clearly, these staging systems would be more familiar to orthopedic surgeons.

Diagnostic imaging has long played a major role in the investigation of suspected osteomyelitis. When interpreting the literature on performance characteristics of different radiographic tests, it is important to remember that osteomyelitis is a heterogeneous condition, and to be clear on what clinical entity the particular modality is assessing (eg, acute or chronic osteomyelitis, presence or absence of neuropathic changes). In a similar vein, it is critical that any individual patient's imaging results be interpreted in light of the clinical situation, considering what patient-specific features may interfere with the test's reported sensitivity and specificity.

A systematic review and meta-analysis of several diagnostic imaging modalities for the assessment of chronic osteomyelitis was published in 2005 (11). The authors selected articles from the literature in which the imaging technique was compared with histology, culture results and clinical follow-up for more than six months. In their review, Termaat et al (11) reported a sensitivity of $60 \%$ (95\% CI 28 to 86 ) and specificity of $67 \%$ (95\% CI 36 to 89 ) for plain radiography (11). To improve on sensitivity, several other imaging modalities have been evaluated for their diagnostic utility. The first of these has been bone scintigraphy with ${ }^{99} \mathrm{~m}$ Tc-diphosphonate. While it is clearly better than plain films in terms of sensitivity (82\%), bone scintigraphy is seriously disadvantaged by its very poor specificity (25\%). Taking advantage of the inflammatory response in osteomyelitis, white blood cell scans have been examined in the diagnosis of chronic osteomyelitis. In the meta-analysis (11), leukocyte scanning had improved specificity (77\%, 95\% CI 63 to 87 ) but poor sensitivity $(61 \%, 95 \%$ CI 43 to 76), lending support to the practice of combining bone and leukocyte scintigraphy to optimize diagnostic capability (11). The low sensitivity of the leukocyte scan was largely driven by its poor performance in axial skeleton osteomyelitis, where the sensitivity was $21 \%$, compared with peripheral skeleton, where the sensitivity was $84 \%$ (11). Combined bone and gallium scanning showed no advantage over bone and white blood cell scanning (11).

Over the past decade, cross-sectional imaging modalities such as computed tomography (CT) and magnetic resonance imaging (MRI) have become popular diagnostic methods, in part because of their high anatomical resolution. CT identifies areas of necrotic bone, soft-tissue infection and altered marrow density due to osteomyelitis (8). However, altered marrow density can also be seen with neoplasms, hemorrhage, fractures or irradiation (12). In the one small study that met Termaat et al's criteria (11), CT had a sensitivity of $67 \%$ (95\% CI 24 to 94 ) and specificity of $50 \%$ (92\% CI 3 to 97 ) in the diagnosis of chronic osteomyelitis of the spine. Where CT can play an important role is in delineating the extent of necrotic bone and thereby guiding debridement (12). In the meta-analysis (five eligible studies), MRI had a sensitivity (84\%) in the same range as that of a bone scan, with somewhat better specificity (60\%, 95\% CI 38 to 78$)$. MRI has been suggested as the

${ }^{1}$ Queen Elizabeth II Health Sciences Centre and Dalhousie University, Halifax, Nova Scotia; ${ }^{2}$ Departments of Medicine, Pathology and

Laboratory Medicine, and Microbiology and Infectious Diseases, University of Calgary, Calgary, Alberta

Correspondence and reprints: Dr BL Johnston, Queen Elizabeth II Health Sciences Centre, 5014ACC - 1278 Tower Road, Halifax, Nova Scotia B3H 2Y9. Telephone 902-473-5553, fax 902-473-7394, e-mail ljohnsto@dal.ca

Received and accepted for publication March 23, 2007 
modality of choice in determining the extent of infection, especially when epidural abscess with or without neural compression is of concern (12). Despite being a relatively new technique, there were four studies on positron emission tomography (PET) in the meta-analysis (11). PET had a sensitivity of 96\% (95\% CI 88 to 99), which was higher than all other modalities. The specificity of PET was lower at 91\% (95\% CI 81 to 95), but this was still an improvement over all other imaging types. Based on this meta-analysis, PET scanning has the highest diagnostic accuracy for confirming or excluding the presence of chronic osteomyelitis (11). Unfortunately, PET is of limited availability in most health care facilities.

Given the less than perfect performance characteristics, the role of diagnostic imaging should be viewed as confirming the presumed clinical diagnosis and providing information regarding the exact site and extent of infection (12). As with most tests, no technique can absolutely confirm or exclude the diagnosis. All results have to be carefully interpreted in light of the clinical findings. As Haas and McAndrew observed (10), imaging studies provide information that supports or refutes the clinical suspicion with variable certainty!

There is agreement that the gold standard for diagnosing osteomyelitis is bone biopsy and culture. This recommendation has some foundation from the literature, dating back to the 1970s. In a study that compared sinus track cultures with bone biopsy obtained at surgery (13), sinus culture was only $44 \%$ sensitive in identifying the etiological organism. A frequently noted positive feature of this study was the good correlation between sinus and bone specimens for Staphylococcus aureus. This has led some to point to the value of sinus culture for diagnosing $S$ aureus osteomyelitis (6). These findings have been supported in part by Perry et al (14). They found that isolating a single organism (usually $S$ aureus) predicted the bone culture result in $90 \%$ of situations. This dropped to $26 \%$ when there was polymicrobial growth. Despite this, there seems to be reluctance on the part of many surgeons to obtain a biopsy specimen. This may relate to overinterpretation of the utility of sinus cultures. More recently, concordance between bone and nonbone specimens was examined in a cohort of patients with chronic osteomyelitis, primarily contiguous and frequently following trauma (15). The overall diagnostic accuracy of nonbone specimens was $30 \%$, increasing to $42 \%$ for $S$ aureus, and $35 \%$ when taken from sinus tracts (15). However, this was no better than chance alone (15). Results from these studies should prompt us to reconsider the rigour with which we pursue an etiological diagnosis of chronic osteomyelitis in most circumstances.

In 1988, Carl Norden (16) reported that optimal therapy for chronic osteomyelitis was unclear, and that neither the critical duration of therapy nor the relative efficacy of various antibiotic classes was known. The situation is no less murky 20 years later, although treatment suggestions can be found in any number of texts and reviews. Treatment guidelines for acute and chronic osteomyelitis in adults recommend prolonged courses of antimicrobials, usually given intravenously. The recommendations for treatment duration for acute osteomyelitis are fairly uniform at four to six weeks. There is more variability in recommendations for chronic osteomyelitis treatment, with differing routes and durations, and sometimes related to the stage of infection, pathogen and intent for cure or long-term suppression of a frequently relapsing infection $(1-4,6-8,10,17,18)$. Surgical debridement is a mainstay of management for chronic osteomyelitis, but is frequently not required in the acute form. The evidence to support the various regimens and durations comes from a variety of sources, including animal and human pharmacokinetic studies, animal studies of the efficacy of various antimicrobials, case reports and series, as well as randomized and nonrandomized trials in humans.

In 1970, Waldvogel et al (1) recommended that hematogenous osteomyelitis be treated with high-dose antibiotics for at least four weeks. The rationale was that hematogenous osteomyelitis represented a septic metastasis, and that penicillin penetration of bone required high doses of antibiotics. They supported this recommendation by describing the outcomes of patients who had received intensive (four weeks or longer) or limited therapy and, incidentally, noted that surgery was not necessarily required. In terms of chronic osteomyelitis, they noted that the outcome of therapy was similar in patients who received intensive and limited antimicrobial treatment, but a pattern of prolonged therapy for chronic osteomyelitis emerged (2). And so, whether right or wrong (and animal studies suggest that prolonged therapy is right), the evidence base for recommending a long course of antibiotics in humans is rather anecdotal in its origins. More analytical studies over the years have generally assumed four to six weeks of intravenous therapy as the gold standard against which new agents or new routes of treatment are measured.

Some clinicians consider selecting a specific antimicrobial based on its reported penetration into bone. Data related to bone penetration come from animal studies in which the bone and serum concentrations of antibiotics used to treat experimentally infected rats or rabbits are measured. These studies generally demonstrate excellent bone penetration by rifampin $(19,20)$ and clindamycin $(21)$, with good penetration by fluoroquinolones (19) and low bone concentrations achieved by cefazolin $(20,21)$. Human pharmacokinetic studies are largely performed in the setting of surgical prophylaxis for joint arthroplasty. From several studies (22-24), it appears that beta-lactams penetrate to a lesser extent into uninfected bone than clindamycin. However, Darley and MacGowan (18) suggest caution in interpreting the results of such bone penetration studies in humans. They note that the methodology has not been standardized, assay procedures may affect results considerably, healthy bone may not reflect pharmacokinetics in infected bone, penetration into cortical and medullary bone differs, and that blood contamination may not have been accounted for.

Glycopeptides, beta-lactams, fluoroquinolones, clindamycin and rifampin have all been studied in an animal model of $S$ aureus osteomyelitis. In one experimental model, clindamycin was superior to cefazolin in eradicating $S$ aureus from infected bone (21). Interestingly, mortality was higher in the clindamycin-treated rabbits in this study (35\% versus $9 \%$; $\mathrm{P}=$ not significant). In another study (20), $45 \%$ of rabbits on rifampin, $67 \%$ on cephalothin and $95 \%$ on trimethoprim were still culture-positive after 28 days of antibiotics. Fewer animals given a combination of cephalothin and rifampin were culturepositive than when given single agent therapy, but when S aureus was isolated, it was uniformly resistant to rifampin. On the other hand, another study in the rabbit model (19) demonstrated a benefit for combinations, including rifampin, in terms of decreasing the number of organisms in bone (although none sterilized bone) without the emergence of rifampin resistance. Limitations of animal studies include lack of debridement, 
high initial inocula, lack of experience with recurrent or prolonged infection and lack of extended follow-up (18). These studies do, however, give a sense of the difficulty involved in curing these infections and the prolonged course of antimicrobials that is required.

What have we learned from clinical trials in humans that evaluate different osteomyelitis treatment modalities? One thing we have learned is that, methodologically, the studies are generally very poor $(25,26)$. Lazzarini et al $(25)$ noted that most studies were noncomparative, and that the comparative trials involved relatively few patients. The associated surgical management is often not described and follow-up is rarely more than 12 months (25). This has also been noted by Stengel et al (26), who were hampered in their meta-analysis of antibiotic therapy for osteomyelitis by small sample sizes, missing descriptions of patient populations and disease characteristics, and the frequent use of concomitant antibiotics. After reviewing hundreds of studies, both groups concluded that the available literature on the treatment of osteomyelitis was inadequate to determine the best agents, route or duration of antibiotic therapy $(25,26)$. In particular, oral fluoroquinolones, with or without rifampin, were no better or worse than standard parenteral treatments (25-28). Oral fluoroquinolones, with their excellent bioavailability, do offer a cost advantage to intravenous treatments and are more convenient to patients. However, in their meta-analysis comparing oral fluoroquinolones with nonfluoroquinolone intravenous comparators, Stengel et al (26) found a significant difference in drug-related side effects favouring the parenteral arm $(\mathrm{P}=0.0002)$, although there was significant heterogeneity among studies. While the debate goes on regarding the role of fluoroquinolones in the treatment of osteomyelitis, they are not listed as the first-line agent for Gram-positive infections $(6-8,17)$. A similar debate is likely to continue with respect to rifampin. There is no doubt about its impressive results in animal studies. In the one randomized (nonblinded) but underpowered study comparing nafcillin with and without rifampin, there was no difference between the two treatment arms (29). Rifampin is not recommended in addition to beta-lactams as first-line therapy for staphylococcal osteomyelitis $(4,6,7,17,18)$.

Contiguous osteomyelitis with vascular involvement, most often involving diabetics, is a commonly observed form of osteomyelitis that exemplifies the challenges in diagnosis and treatment of chronic bone infections. In one study, the majority of diabetic foot ulcers $(68 \%)$ had an underlying osteomyelitis that was infrequently (32\%) diagnosed clinically (30). They also found that all patients with ulcers that exposed bone had osteomyelitis (30). Palpation of bone in the infected foot ulcer of a diabetic patient has $66 \%$ sensitivity and $85 \%$ specificity, and is a reasonable initial assessment tool (31). Diagnostic imaging presents a particular challenge in the neuropathic foot (32), even with MRI (33). Indeed, in one decision and costeffectiveness analysis (32), it was suggested that noninvasive testing adds significant expense to the treatment of diabetic patients with pedal osteomyelitis and may offer little improvement in outcome. Most literature suggests that ulcer swabs correlate poorly with tissue specimens in this population (34-36), although there is debate as to whether treatment based on bone biopsy results will improve the clinical outcome and prognosis compared with broad-spectrum empirical treatment or treatment based on swab culture results $(32,36,37)$. The treatment studies for contiguous osteomyelitis with vascular involvement are of a similar quality to those for osteomyelitis in general (26). In fact, they are often one and the same, with diabetics not infrequently representing the larger cohort of patients in these trials. Despite these limitations in evidence, the Infectious Diseases Society of America has published a guideline for the diagnosis and treatment of diabetic foot infections (38). They state that highly bioavailable oral antibiotics can be used in some cases of osteomyelitis, and that at least four to six weeks of therapy is required, and probably longer if infected bone remains (38).

So, where does that leave us in terms of managing chronic osteomyelitis? For now, it appears that the art of medicine will continue to play a role in diagnosis and treatment, with science providing some foundation. Even with the recognition that osteomyelitis is a heterogeneous infection, it is difficult to believe that better-quality human trials are not available to guide investigation and treatment. While trials assessing different antimicrobials are unlikely to offer new insights, effectiveness studies evaluating culture-directed versus empirical regimens, differing treatment durations and the precise role for surgical intervention for chronic osteomyelitis would hopefully provide for better-reasoned approaches to management.

\section{REFERENCES}

1. Waldvogel FA, Medoff G, Swartz MN. Osteomyelitis: A review of clinical features, therapeutic considerations and unusual aspects. N Engl J Med 1970;282:198-206.

2. Waldvogel FA, Medoff G, Swartz MN. Osteomyelitis: A review of clinical features, therapeutic considerations and unusual aspects. N Engl J Med 1970;282:260-6.

3. Waldvogel FA, Papageorgiou PS. Osteomyelitis: The past decade. N Engl J Med 1980;303:360-70

4. Lew DP, Waldvogel FA. Osteomyelitis. N Engl J Med 1997;336:999-1007.

5. Calhoun JH, Manring MM. Adult osteomyelitis. Infect Dis Clin North Am 2005;19:765-86.

6. Berbari EF, Steckelberg JM, Osmon DR. Osteomyelitis. In: Mandell GL, Bennett JE, Dolin R, eds. Principles and Practice of Infectious Diseases. Philadelphia: Elsevier Inc, 2005:1322-32.

7. Gilbert DN, Moellering RC, Eliolopoulos GM, Sande MA. The Sanford Guide to Antimicrobial Therapy 2006. Sperryville: Antimicrobial Therapy, Inc, 2006:4-5,52.

8. Mader JT, Shirtliff M, Calhoun JH. Staging and staging application in osteomyelitis. Clin Infect Dis 1997;25:1303-9.

9. Cierny G 3rd, Mader JT, Penninck JJ. A clinical staging system for adult osteomyelitis. Clin Orthop Relat Res 2003;414:7-24.

10. Haas DW, McAndrew MP. Bacterial osteomyelitis in adults: Evolving considerations in diagnosis and treatment. Am J Med 1996;101:550-61.

11. Termaat MF, Raijmakers PG, Scholten HJ, Bakker FC, Patka P, Haarman HJ. The accuracy of diagnostic imaging for the assessment of chronic osteomyelitis: A systemic review and metaanalysis. J Bone Joint Surg Am 2005;87:2464-71.

12. Pineda C, Vargas A, Rodriguez AV. Imaging of osteomyelitis: Current concepts. Infect Dis Clin North Am 2006;20:789-825.

13. Mackowiak PA, Jones SR, Smith JW. Diagnostic value of sinustract cultures in chronic osteomyelitis. JAMA 1978;239:2772-5.

14. Perry CR, Pearson RL, Miller GA. Accuracy of cultures of material from swabbing of the superficial aspect of the wound and needle biopsy in the preoperative assessment of osteomyelitis. J Bone Joint Surg Am 1991;73:745-9.

15. Zuluaga AF, Galvis W, Saldarriaga JG, Agudelo M, Salazar BE, Vesga O. Etiologic diagnosis of chronic osteomyelitis. Arch Intern Med 2006;166:95-100.

16. Norden CW. Lessons learned from animal models of osteomyelitis. Rev Infect Dis 1988;10:103-10.

17. Davis JS. Management of bone and joint infections due to Staphylococcus aureus. Intern Med J 2005;35(Suppl 2):S79-96.

18. Darley ES, MacGowan AP. Antibiotic treatment of Gram-positive bone and joint infections. J Antimicrob Chemother 2004;53:928-35. 
19. Dworkin R, Modin G, Kunz S, Rich R, Zak O, Sande M. Comparative efficacies of ciprofloxacin, pefloxacin, and vancomycin in combination with rifampin in a rat model of methicillin-resistant Staphylococcus aureus chronic osteomyelitis. Antimicrob Agents Chemother 1990;34:1014-6.

20. Norden CW. Experimental chronic staphylococcal osteomyelitis in rabbits: Treatment with rifampin alone and in combination with other antimicrobial agents. Rev Infect Dis 1983;(Suppl 3):S491-4.

21. Mader JT, Adams K, Morrison L. Comparative evaluation of cefazolin and clindamycin in the treatment of experimental Staphylococcus aureus osteomyelitis in rabbits. Antimicrob Agents Chemother 1989;33:1760-4

22. Nicholas P, Meyers BR, Levy RN, Hirschman SZ. Concentration of clindamycin in human bone. Antimicrob Agents Chemother 1975;8:220-1.

23. Fitzgerald RH Jr, Kelly PJ, Snyder RJ, Washington JA II. Penetration of methicillin, oxacillin, and cephalothin into bone and synovial tissues. Antimicrob Agents Chemother 1978;14:723-6.

24. Smilack JD, Flittie WH, Williams TW Jr. Bone concentrations of antimicrobial agents after parenteral administration. Antimicrob Agents Chemother 1976;9:169-71.

25. Lazzarini L, Lipsky BA, Mader JT. Antibiotic treatment of osteomyelitis: What have we learned from 30 years of clinical trials? Internat J Infect Dis 2005;9:127-38.

26. Stengel D, Bauwens K, Sehouli J, Ekkernkamp A, Porzsolt F. Systematic review and meta-analysis of antibiotic therapy for bone and joint infections. Lancet Infect Dis 2001;1:175-88.

27. Gentry LO, Rodriguez GG. Oral ciprofloxacin compared with parenteral antibiotics in the treatment of osteomyelitis. Antimicrob Agents Chemother 1990;34:40-3.

28. Gentry LO, Rodriguez-Gomez G. Ofloxacin versus parenteral therapy for chronic osteomyelitis. Antimicrob Agents Chemother 1991;35:538-41.
29. Norden CW, Bryant R, Palmer D, Montgomerie JZ, Wheat J. Chronic osteomyelitis caused by Staphylococcus aureus: Controlled clinical trial of nafcillin therapy and nafcillin-rifampin therapy. South Med J 1986;79:947-57.

30. Newman LG, Waller J, Palestro CJ, et al. Unsuspected osteomyelitis in diabetic foot ulcers. Diagnosis and monitoring by leukocyte scanning with Indium In 111 oxyquinoline. JAMA 1991;266:1246-51.

31. Grayson ML, Gibbons GW, Balogh K, Levin E, Karchmer AW. Probing to bone in infected pedal ulcers. A clinical sign of underlying osteomyelitis in diabetic patients. JAMA 1995;273:721-3.

32. Eckman MH, Greenfield S, Mackey WC, et al. Foot infections in diabetic patients. Decision and cost-effectiveness analyses. JAMA 1995;273:712-20.

33. Ahmadi ME, Morrison WB, Carrino JA, Schweitzer ME, Raikin SM, Ledermann HP. Neuropathic arthropathy of the foot with and without superimposed osteomyelitis: MR imaging characteristics. Radiology 2006;238:622-31.

34. Sapico FL, Canawati HN, Witte JL, et al. Quantitative aerobic and anaerobic bacteriology of infected diabetic feet. J Clin Microbiol 1980;12:413-20.

35. Senneville E, Melliez H, Beltrand E, et al. Culture of percutaneous bone biopsy specimens for diagnosis of diabetic foot osteomyelitis: Concordance with ulcer swab cultures. Clin Infect Dis 2006;42:57-62.

36. Wheat LJ, Allen SD, Henry M, et al. Diabetic foot infections. Bacteriologic analysis. Arch Intern Med 1986;146;1935-40.

37. Embil JM, Trepman E. Microbial evaluation of diabetic foot osteomyelitis. Clin Infect Dis 2006;42:63-5.

38. Lipsky BA, Berendt AR, Deery HG, et al; Infectious Diseases Society of America. Diagnosis and treatment of diabetic foot infections. Clin Infect Dis 2004;39:885-910. 


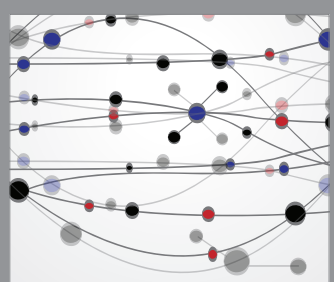

The Scientific World Journal
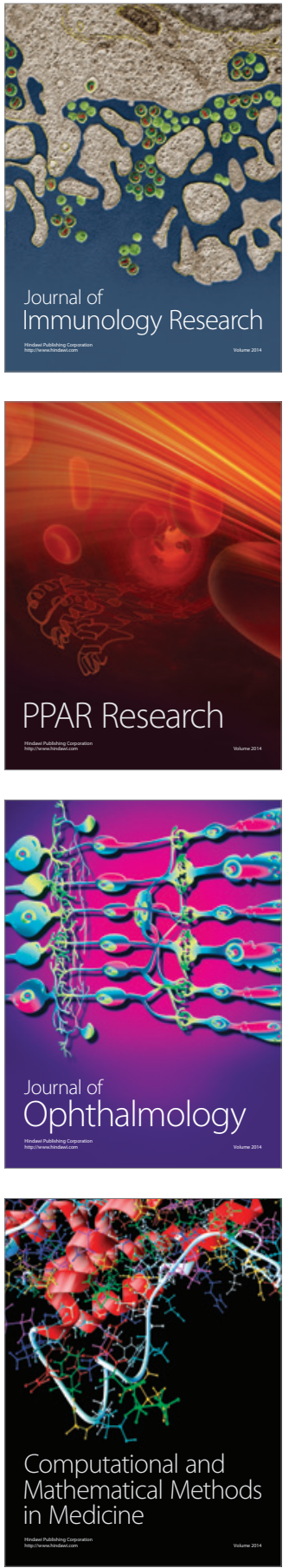

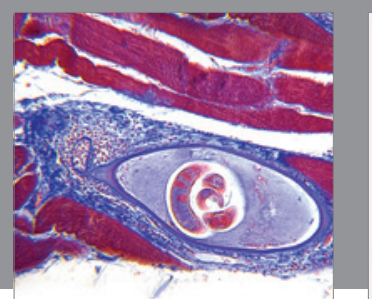

Gastroenterology Research and Practice

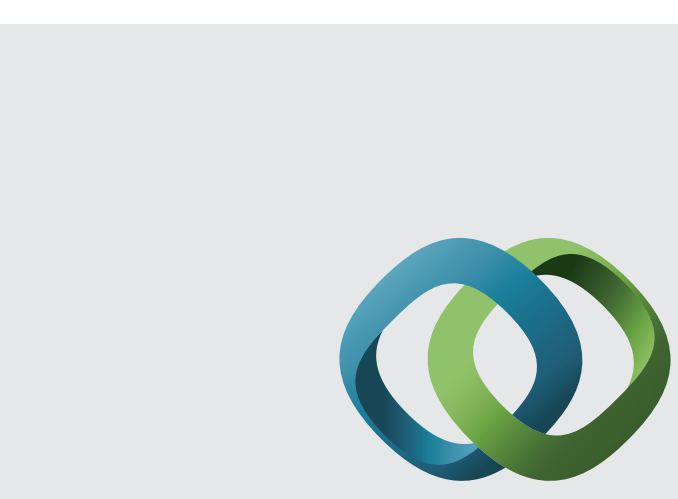

\section{Hindawi}

Submit your manuscripts at

http://www.hindawi.com
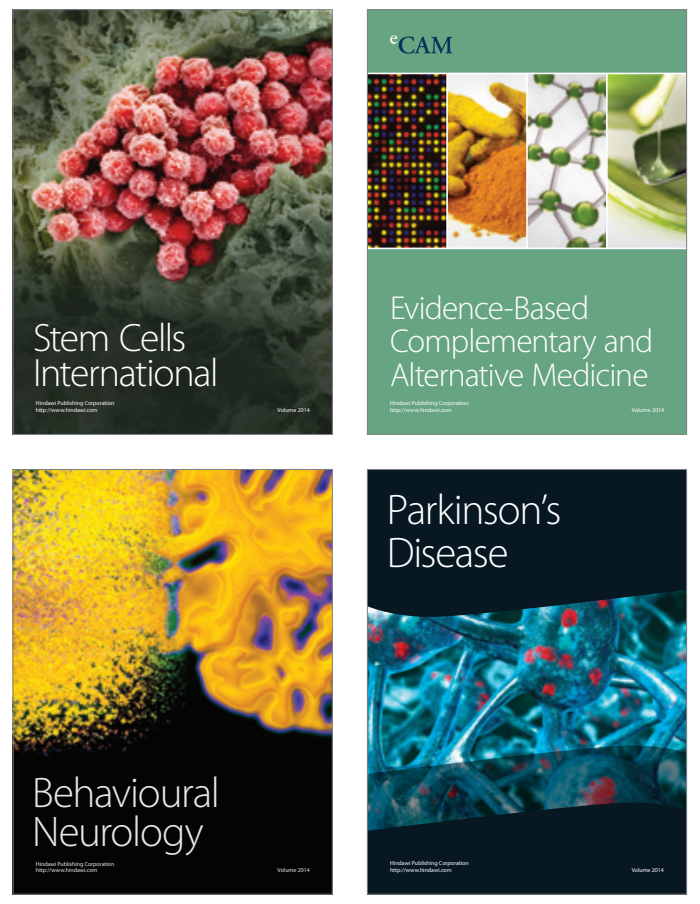
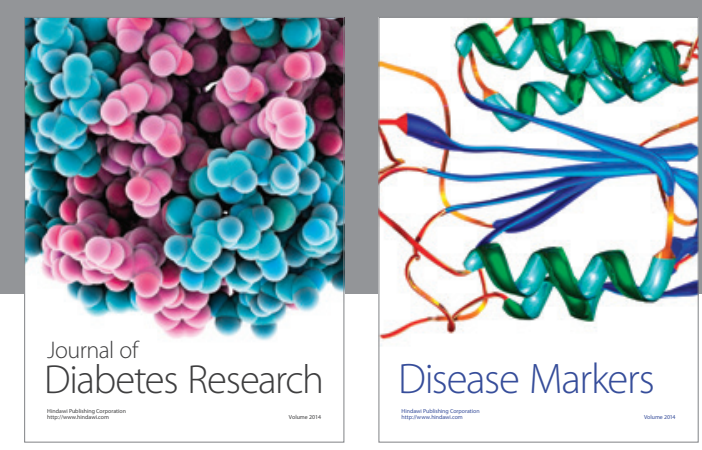

Disease Markers
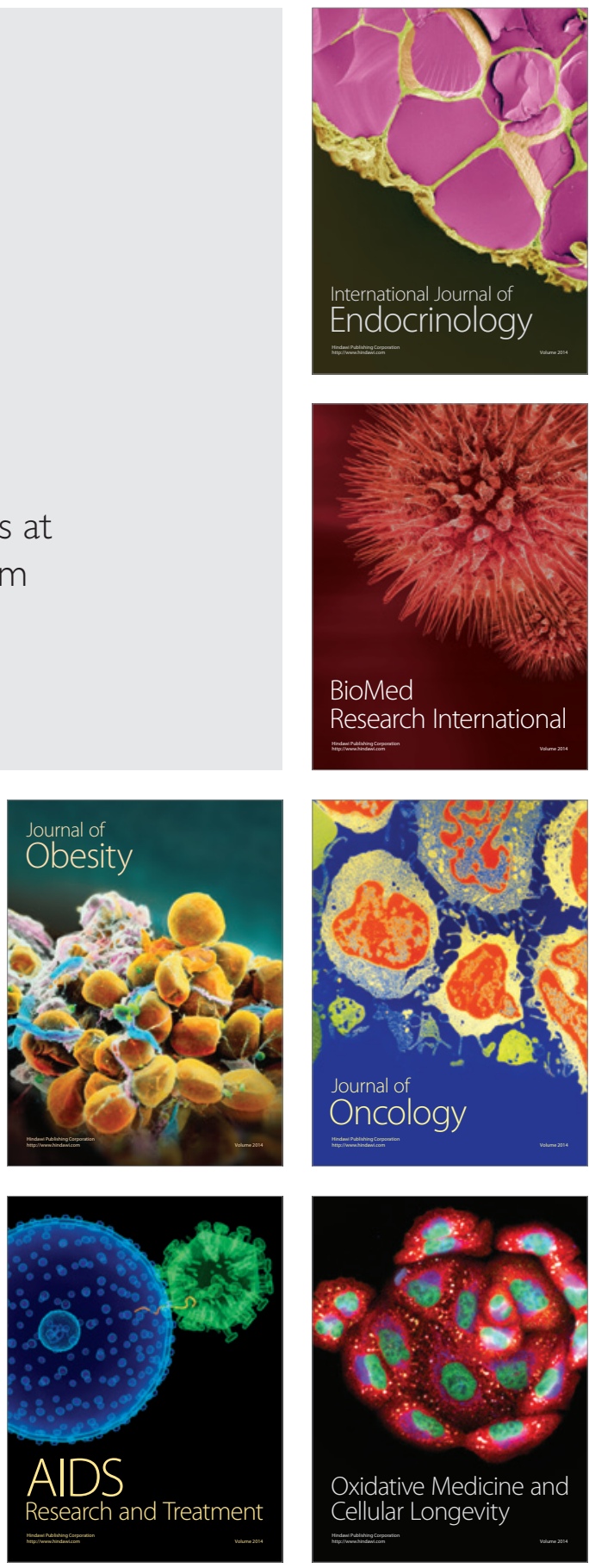